\title{
Long-term Outcomes of Index Cryo-balloon ablation in Patients with Atrial Fibrillation and Systolic Heart Failure
}

\author{
${ }^{1}$ Alfred Hospital \\ ${ }^{2}$ Saint Bartholomew's Hospital Barts Heart Centre \\ ${ }^{3}$ Barts Health NHS Trust \\ ${ }^{4}$ Baker Heart and Diabetes Institute \\ ${ }^{5}$ Barts Heart Centre, Barts Health NHS trust \\ ${ }^{6}$ Barts Heart Centre \\ ${ }^{7}$ Barts Heart Centre, St Bartholomew's Hospital \\ ${ }^{8}$ Bart's Heart Centre \\ ${ }^{9}$ Barts and The London NHS Trust \\ ${ }^{10}$ St Bartholomew's Hospital
}

Sandeep Prabhu ${ }^{1}$, Nikhil Ahluwalia ${ }^{2}$, Sara Tyebally ${ }^{2}$, Adam Dennis ${ }^{2}$, Samuel Malomo ${ }^{2}$, Aderonke Abiodun ${ }^{2}$, Agelos Tyrlis ${ }^{2}$, Gurpreet Dhillon ${ }^{3}$, Louise Segan ${ }^{4}$, Adam Graham ${ }^{3}$, Shohreh Honarbakhsh ${ }^{5}$, Vinit Sawhney ${ }^{6}$, Simon Sporton ${ }^{7}$, Martin Lowe ${ }^{3}$, Malcolm Finlay ${ }^{3}$, Mark Earley ${ }^{3}$, Pier Lambiase ${ }^{8}$, Richard Schilling 9 , and Ross Hunter ${ }^{10}$

October 8, 2020

\begin{abstract}
Background: Although catheter ablation $(\mathrm{CA})$ is an effective treatment for patients with AF and LVSD, the efficacy of an initial cryo-ablation strategy is unknown. This study evaluated long term outcomes of patients with an initial cryo-ablation strategy for AF and LV systolic dysfunction (LVSD). Methods: Outcomes of patients undergoing index cryoablation for AF from January 2008 until March 2018, with documented pre-ablation LVEF?45\% were evaluated for long term freedom from AF and change in ventricular function from baseline. Results: 76 patients met inclusion criteria. Patients were predominantly male (80\%), aged 63 ? 11yrs, with an average CHADS2 VASc score of 2.66 ? 1.40, baseline LVEF 34 ? $8.7 \%$ (ischaemic in $37 \%$ ) and NYHA class 2.37 ? 0.72 . Repeat procedures were performed in $13 \%$. The single procedure success was $70 \%$ at 1 year $(81 \%$ for paroxysmal AF, $65 \%$ for persistent AF, $29 \%$ for long-standing persistent AF) and at 28 ? 13 months follow up was $43 \%$ (50\%, $41 \%$ and $21 \%$ respectively), increasing to $59 \%$ allowing for subsequent redo procedure with RF ablation $(64 \%, 57 \%$, and $50 \%$ respectively). At follow up, LVEF significantly improved to 46 ? 16\% ( $<<0.001)$, and NYHA reduced to $1.51 ? 0.66$ (p<0.001).

Conclusion: An initial strategy of cryoablation in patients with AF and LVSD is an effective approach in the treatment of patients with concurrent AF and LVSD. Larger, randomised prospective studies are required to confirm these findings.
\end{abstract}

\section{Title:}

Long-term Outcomes of Index Cryo-balloon ablation in Patients with Atrial Fibrillation and Systolic Heart Failure

Brief Title

Cryoablation in Heart Failure

Word Count: 4428 


\section{Authors}

Prabhu $\mathrm{S}^{1,2,3 *}$, Ahluwalia $\mathrm{N}^{1 *}$, Tyebally $\mathrm{SM}^{1}$, Dennis $\mathrm{ASC}^{1}$, Malomo $\mathrm{SO}^{1}$, Abiodun $\mathrm{AT}^{1}$, Tyrlis $\mathrm{A}^{1}$, Dhillon $\mathrm{G}^{1}$, Segan, $\mathrm{L}^{2}$, Graham $\mathrm{A}^{1}$, Honarbakhsh $\mathrm{S}^{1}$, Sawhney $\mathrm{V}^{1}$, Sporton $\mathrm{S}^{1}$, Lowe $\mathrm{M}^{1}$, Finlay $\mathrm{M}^{1}$, Earley $\mathrm{MJ}^{1}$, Lambiase $\mathrm{P}^{1}$, Schilling RJ ${ }^{1}$, Hunter RJ ${ }^{1}$.

${ }^{*}$ Co-first authors

\section{Affiliations}

${ }^{1}$ Department of Cardiology, St Bartholomew's Hospital, London, UK

${ }^{2}$ Baker Heart and Diabetes Research Institute, Melbourne, Australia.

${ }^{3}$ University of Melbourne, Melbourne, Australia.

\section{Address for Correspondence}

Dr. Sandeep Prabhu

Cardiologist, Electrophysiologist and Clinical Research Fellow

Barts Heart Centre, The Alfred Hospital and Baker Heart and Diabetes Institute

S.Prabhu@alfred.org.au

Telephone: +61433172620

\section{Structured Abstract}

\section{Background :}

Although catheter ablation (CA) is an effective treatment for patients with AF and LVSD, the efficacy of an initial cryo-ablation strategy is unknown. This study evaluated long term outcomes of patients with an initial cryo-ablation strategy for AF and LV systolic dysfunction (LVSD).

\section{Methods :}

Outcomes of patients undergoing index cryoablation for AF from January 2008 until March 2018, with documented pre-ablation LVEF[?] $45 \%$ were evaluated for long term freedom from AF and change in ventricular function from baseline.

\section{Results :}

76 patients met inclusion criteria. Patients were predominantly male $(80 \%)$, aged $63 \pm 11$ yrs, with an average $\mathrm{CHADS}_{2}$ VASc score of $2.66 \pm 1.40$, baseline LVEF $34 \pm 8.7 \%$ (ischaemic in 37\%) and NYHA class $2.37 \pm$ 0.72 . Repeat procedures were performed in $13 \%$. The single procedure success was $70 \%$ at 1 year $(81 \%$ for paroxysmal AF, $65 \%$ for persistent AF, $29 \%$ for long-standing persistent AF) and at $28 \pm 13$ months follow up was $43 \%(50 \%, 41 \%$ and $21 \%$ respectively), increasing to $59 \%$ allowing for subsequent redo procedure with RF ablation (64\%, $57 \%$, and $50 \%$ respectively). At follow up, LVEF significantly improved to $46 \pm 16 \%$ $(\mathrm{p}<0.001)$, and NYHA reduced to $1.51 \pm 0.66(\mathrm{p}<0.001)$.

\section{Conclusion :}

An initial strategy of cryoablation in patients with AF and LVSD is an effective approach in the treatment of patients with concurrent AF and LVSD. Larger, randomised prospective studies are required to confirm these findings.

\section{Keywords}

Atrial fibrillation 
Heart failure

Cryoablation

\section{Condensed abstract}

Catheter ablation is an established, effective approach for the treatment of AF in patients with heart failure, however the role of cryoablation in this setting is unclear. Procedural success and LVEF improvement in patients with LVEF[?]45\% undergoing index catheter ablation with cryoablation were evaluated. Freedom from $\mathrm{AF}$ recurrence was seen in $43 \%$ rising to $59 \%$ following repeat procedure. There were significant improvements in LVEF and functional status at long-term follow up. Cryoablation is an effective first-line $\mathrm{AF}$ ablation approach in the setting of heart failure.

\section{Abbreviation list}

CFAE complex fractionated atrial electrograms

CRYO cryoablation

CTI cavo-tricuspid isthmus

HF heart failure

LVEF left ventricular systolic dysfunction

PV pulmonary vein

$\mathrm{RF}$ radio-frequency

\section{Introduction}

Atrial fibrillation (AF) and heart failure are both emerging epidemics and frequently occur together ${ }^{1-3}$. Whilst previously considered a passive bystander in the setting of heart failure, AF is now recognised as an active determinant of clinical outcome $\mathrm{e}^{4,5}$ and not infrequently, the primary driver of the heart failure itself $^{6,7}$. Recently, catheter ablation of AF has established itself as an effective approach to the restoration of sinus rhythm in patients with heart failure without reliance upon long term anti-arrhythmic therapy, with several randomised studies showing improvements in ventricular function ${ }^{6,8,9}$, symptoms ${ }^{10}$, reduced hospitalisations and mortality ${ }^{8}$.

Although cryoablation has rapidly evolved as a mainstream treatment for AF in the paroxysmal phenotype, its role in the setting of persistent AF and particularly in those with structural heart disease remains largely unexplored. Given that there is limited evidence for a lesion set beyond pulmonary vein isolation at the index procedure, cryoablation may be a feasible first line therapy in this cohort. We report long-term outcomes in patients with heart failure undergoing index ablation with cryoablation.

\section{Methods}

\section{Study population}

This was a retrospective, single centre analysis of consecutive patients with systolic heart failure undergoing catheter ablation for AF at the Barts Heart Centre from $1^{\text {st }}$ January 2008 to $30^{\text {th }}$ March 2018. Patients were included if they: (1) had documented pre-procedural LVEF [?]45\% as determined by either echocardiography or cardiac MR within 6 months of the index procedure; (2) underwent index catheter ablation for AF incorporating de-novo pulmonary vein isolation with cyroballoon (CRYO). Patients were excluded if (1) they had $<12$ months of follow up; (2) had previous left atrial ablation for AF; (3) if clinical data was incomplete. This study was approved by the hospital's relevant governing body (clinical effectiveness unit approval number: 10573).

Cryoablation Procedure 
Anticoagulation was continued uninterrupted for all patients. Procedures were performed under conscious sedation or general anaesthesia. Single transeptal puncture was performed. Ablation was performed using 28mm Arctic Front Advance Cryoballoon System (Medtronic) via a 14F FlexCath Cryosheath (Medtronic, Ireland). Each PV was engaged with a multi-polar catheter (Achieve wire, Medtronic) and with demonstration of PV electrograms where possible. After inflation, pulmonary venous occlusion was confirmed with venography, and freezes between 180-240 seconds applied to each vein, aiming for abolition of PV signals and freeze temperatures between $-40 \mathrm{degC}$ to $-55 \mathrm{degC}$. Freezes for the right PVs were performed with simultaneous phrenic nerve stimulation from high output pacing via a Quad catheter positioned in the right subclavian vein. A single good freeze was considered sufficient if it isolated a pulmonary vein, but further freezes were deployed if a vein failed to isolate, or at operator discretion if the freeze was deemed poor. Patients remaining in $\mathrm{AF}$ at procedure end were electrically cardioverted to sinus rhythm. In the case of documented CTI dependant atrial flutter, CTI ablation was also performed using an irrigated RF catheter (without a 3D mapping system or contact force sensing technology).

\section{Follow up}

Antiarrhythmic medications were continued for a minimum of 3 months post ablation and then weaned or ceased at the discretion of the treating physician. Follow-up included clinical assessment and 12 lead ECG, at 3,6 and 12 months and then ongoing follow-up as dictated by symptoms. Patients with implantable cardiac devices capable of AF detection (dual chamber device or implantable loop recorders) had device interrogation performed at 3 months then yearly as a minimum. Patients without an intracardiac device underwent 48 hours of ambulatory monitoring at either 3 or 6 months follow-up with further monitoring dictated by symptoms. Recurrence was defined as documented AF/AT $>30$ seconds with or without clinical symptoms, or recurrence requiring pharmacological or interventional treatment, beyond a 3 month post procedural blanking period. The ongoing use of antiarrhythmic drug therapy was not counted as failure (since this was not part of a trial with a protocol to stop them necessarily) but the success rate is reported on and off antiarrhythmic drugs. Information was obtained from the hospital data-registry and verified by assessment of hospital medical records. Cardiac specific beta-blockers specifically used for heart failure therapy were not classified as anti-arrhythmic agents. Repeat ablation was offered to patients with symptomatic recurrence as clinically indicated and was performed exclusively with RF ablation. The ablation strategy involved PV re-isolation followed by mapping of induced or spontaneous atrial tachycardias and further substrate-based ablation at the operator discretion.

Statistical analysis

Data are expressed as mean +- standard deviation (SD) unless otherwise indicated. Cox regression analysis was utilised to assess the association of continuous and categorical variables with single and multi-procedure freedom from AF in univariate and multivariate models. A two-tailed p value of $<0.05$ was considered significant. Analyses were conducted using SPSS software (version 26, IBM, Chicago, Illinois).

\section{Results}

Study population

353 patient records were assessed for inclusion (277 were excluded: 130 due to index RF procedure, 58 for LVEF $>45 \%$ and 42 for follow-up data unavailable for [?] $1 \mathrm{yr}, 47$ for previous LA ablation for AF. 76 patients were included for analysis. Baseline characteristics are shown in Table 1. Patients were predominately male (80\%) with an average age of 63 years and $\mathrm{CHADS}_{2}$ VASc risk score of 2.7 with an average LVEF of $34 \%$. A majority had persistent $\mathrm{AF}$ (71\%), of which $27 \%$ had long standing persistent $\mathrm{AF}>1$ year. Most patients had non-ischaemic cardiomyopathy (47\%). Patients were on established anti-heart failure medical therapy with $91 \%$ on RAAS inhibition and $78 \%$ on beta-blocker therapy. The average follow up time was $28+-13$ months.

Procedural characteristics (Table 2)

Isolation of all 4 pulmonary veins was achieved in 75/76 (99\%) of patients. Cavo-tricuspid isthmus (CTI) 
ablation was additionally performed in 14/76 (18\%) of patients. Patients averaged 1.17 procedures over the follow up period with 90 +- 42 minutes per index procedure. Repeat procedures with RF were performed in 10 patients $(13 \%)$. Of those patients $9(90 \%)$ underwent PV re-isolation, two patients (20\%) had additional roof line, one patient (10\%) had complete posterior wall isolation, and two patients (20\%) had additional ablation of complex electrograms.

\section{Complications (Table 3)}

There were no procedure related deaths at the index procedure. There were 3 deaths in the cohort. One patient died from an atrio-oesophageal fistula following redo RF based procedure. The other two patients dies from malignancy at 6 and 9 months post procedure. Vascular complications were seen in two patients (2.2\%), and there were no other reported complications including no stroke (within 30 days), cardiac tamponade or phrenic nerve injury.

Long term outcome (Table 4)

Success following cryoablation was in seen in $43 \%$ (30\% off anti-arrhythmic medications), increasing to $59 \%$ (40\% off anti-arrhythmic medications) with a subsequent redo procedure with $\mathrm{RF}$ ablation, at final follow up. There was no significant difference in success following cryoablation in those patients with paroxysmal $\mathrm{AF}(52 \%)$ compared with persistent $\mathrm{AF}(41 \%, \mathrm{p}=0.36)$ at long term follow up (Figure 1). Twelve month overall freedom from atrial arrhythmia overall was $70 \%$ after a single procedure. Table 4 outlines procedure success at 1 year and final follow up stratified by AF phenotype.

A cox regression analysis was performed incorporating multiple variables including age, gender, hypertension, AF phenotype, heart failure aetiology, NYHA class, LVEF, LA diameter, and $\mathrm{CHADS}_{2}$ VASc score to determine their impact upon long term single and multiple procedure success. There were no significant predictors of single or multi-procedure success (Table 1 supplementary tables).

Heart failure outcomes (Figure 1)

After an average of $18+-16$ months, average left ventricular ejection fraction improved significantly from pre ablation to post ablation $(33+-9.3 \%$ to $46+-16 \%, \mathrm{p}<0.001)$. The average improvement was significantly greater in those patients with non-ischaemic aetiology $(+13+-16 \%)$ versus those with ischaemic cardiomyopathy $(+7.1+-14 \%, \mathrm{p}=0.001)$. Patients demonstrated a significant improvement in average NYHA class at follow up compared to baseline $(2.37+-0.71$ to $1.51+-0.66, \mathrm{p}<0.001)$. Only $10 \%$ of patients were NYHA class I at baseline compared to $58 \%$ at final follow up $(\mathrm{p}<0.001)$. There was no difference in single procedure success in patients with LVEF <35\% (35\%) compared to those with LVEF [?]35\% (47\%, p=0.38). Twenty-seven $(36 \%)$ of patients improved LVEF from $<35 \%$ at baseline to [?]35\% at final follow-up whilst 24 patents $(32 \%)$ had normalised LVEF (>50\%) at final follow up.

\section{Discussion}

To our knowledge, this is the first study to evaluate the long term outcomes of patients with heart failure and atrial fibrillation undergoing catheter ablation with an index cryo-ablation strategy, and the largest reported series to date. The primary findings were:

1. Cryoablation was an effective index approach with long term single and multi-procedure freedom from atrial arrhythmia comparable to contemporary studies of patients with heart failure undergoing RF ablation.

2. Cryoablation in patients with heart failure was safe with relatively low complications rates.

3. Cryoablation is an effective index strategy in improving ventricular function and functional capacity in the setting of $\mathrm{AF}$ and heart failure.

Catheter ablation in heart failure

In recent years, a rapid succession of randomised clinical trials have established the role of catheter ablation as an effective anti-heart failure treatment in patients with concurrent AF and systolic dysfunction ${ }^{6,8-12}$. 
Benefits have included improved quality of life, functional capacity ${ }^{10}$, ejection fraction ${ }^{6}$, reverse remodelling (both atrial ${ }^{13}$ and ventricular ${ }^{14}$ ), reduced hospitalisations ${ }^{8}$ and reduced mortality ${ }^{8,12}$. However, these trials have exclusively utilised RF as the ablation strategy of choice. Whilst there is an increasing evidence base for the efficacy of cryoballoon ablation compared to RF ablation, in patients with paroxysmal AF and the absence of structural heart disease ${ }^{15}$, data regarding the use of cryoablation in the setting of AF and concurrent structural heart disease, and in particular in patients with systolic heart failure, is limited. Pruszkowska et al demonstrated the feasibility of cryoballoon ablation in 30 consecutive patients with LVEF [?] $40 \%$ and continuous monitoring with CIEDs, and demonstrated improvements in NYHA class, EHRA class, AF burden and LVEF, compared to controls undergoing cryoablation without LV dysfunction ${ }^{16}$. Providencia et alcompared outcomes in patients with HF undergoing catheter ablation to those with structurally normal hearts from a multicentre registry and reported use of the cryoballoon in a significant proportion in both groups, suggesting the technique is used in these patients in clinical practice ${ }^{17}$. The present study is the first to demonstrate the efficacy in controlling AF over the long term with an index cryoballoon strategy. The success rates were, as expected, better for paroxysmal AF than for persistent and long standing persistent $\mathrm{AF}$, with results similar to that reported for ablation with radiofrequency ablation in this setting. However, the multivariate analysis did not suggest that any particular group from this cohort fared particularly poorly with cryoablation.

This is also the first study to report on improving ventricular function following AF ablation with an index cryoablation strategy. The greater magnitude of improvement seen in patients with non-ischaemic cardiomyopathy compared to those with ischaemic $(+13 \%$ vs $+7 \%, \mathrm{p}<0.001)$ is consistent with the findings of recent studies, suggesting that absence of fibrosis ${ }^{6}$ or structural heart disease $^{7}$ can predict the extent of LV recovery. We await the outcome of a large multicentre study (CONTRA-HF; NCT03062241) which will directly compare cryoablation to medical therapy in patients with heart failure.

\section{Beyond PVI in the setting of heart failure}

The vast majority of AF in patients with concurrent $\mathrm{LV}$ dysfunction is persistent, and the proportion seen in this study $(71 \%)$ mirrors that in other large studies ${ }^{8,17}$. Given its nature, cryoablation usually entails a PVI only approach to AF ablation. The findings of this study suggests that a PVI-alone approach is an effective ablation strategy in this setting, and that additional index substrate-based ablation may not be necessary to achieve the anti-heart failure treatment effect of catheter ablation. This finding is consistent with that published by Voskoboinik et al which showed that an index PVI only based approach (with either $\mathrm{RF}$ based or cryoablation) in patients with persistent AF (albeit in the absence of structural heart disease) was associated with a 12-month arrhythmia free survival rate of $66.7 \%^{18}$. This is similar to the results seen in other studies ${ }^{19,20}$. Additionally, the improvement in ventricular function despite these modest 'success' rates of $40-60 \%$, highlights the important fact that standard measures of recurrence (AF/AT > 30 secs) may be of little relevance when evaluating the long-term effect of catheter ablation upon improving ventricular function. In this regard, AF burden may be a more useful measure ${ }^{21}$. Thus, arguably, any improved freedom from AF potentially attributable to additional ablation beyond PVI from an RF based approach may not be necessary to achieve the heart failure benefit of catheter ablation. Importantly, repeat procedures were uncommon (9\%) and where performed, PV re-isolation alone was the ablation strategy in the large majority $(71 \%)$ of patients. It should be noted that to date, no substrate modification strategy in persistent AF has shown to benefit outcomes when evaluated in a prospective randomised fashion ${ }^{22}$. This is consistent with the results of a recent meta-analysis suggesting that outcomes of a PVI approach were no different to an approach incorporating linear lesions and ablation of fractionated electrograms ${ }^{5}$,

Procedural considerations

An index CRYO approach may offer several advantages over RF based index ablation. Compared to RF ablation, Cryoablation is generally considered a simplistic and more streamlined and standardized approach to $\mathrm{AF}$ ablation, requiring fewer resources ${ }^{23}$ and an arguably a less steep learning curve compared to RF ablation. The effectiveness of this ablation approach in patients with heart failure, may help to expand access to catheter ablation beyond specialised tertiary centres, potentially facilitating earlier treatment. 
Despite the complexity of patients in this cohort (average $\mathrm{CHADS}_{2} \mathrm{VASC}$ score of 2.7 , and average LVEF of $34 \%$, coronary disease in over a third) - patients had a significant improvement in ventricular function and heart failure symptoms ${ }^{5}$. The shorter more streamlined approach offered by cryoablation may potentially offer an advantage in the heart failure population.

Limitations

There are several important limitations to note. This is a retrospective analysis and is therefore inherently subject to selection bias which may have impacted the results and patient selection. In addition, the effect of interventions such as revascularisation or up-titration of anti-heart failure medical therapy could not be controlled for. A prospective evaluation of cryoablation outcomes in heart failure, including a comparison with RF ablation, should ideally be the focus of prospective randomised studies. It is hoped that these data might inform the design of such trials and provide a limited evidence base for those considering utilizing this approach clinically. The cut-off value of LVEF $<45 \%$ may have introduced selection bias towards less severe phenotypes of heart failure, which may limit the applicability of the findings to patients with more severe LV dysfunction. Ongoing cardiac specific beta-blockade used as anti-failure therapy may have impacted upon AF recurrence.

\section{Conclusion}

Cryoablation is an effective index AF ablation approach in patients with LV systolic dysfunction with comparable results to index $\mathrm{RF}$ ablation with respect to single and multi-procedural freedom from AF and improvement in LV function and functional capacity. Cryoablation is a viable alternative to RF for index ablation in patients with concurrent AF and systolic dysfunction.

\section{References}

1. Lund LH, Savarese G. Global Public Health Burden of Heart Failure. Cardiac Failure Review 2017;03 (01).

2. Chugh SS, Roth GA, Gillum RF, Mensah GA. Global burden of atrial fibrillation in developed and developing nations. Glob Heart 2014;9 (1):113-9.

3. Ling LH, Kistler PM, Kalman JM, Schilling RJ, Hunter RJ. Comorbidity of atrial fibrillation and heart failure. Nat Rev Cardiol 2016;13 (3):131-47.

4. Turagam MK, Garg J, Whang W, Sartori S, Koruth JS, Miller MA, Langan N, Sofi A, Gomes A, Choudry S, Dukkipati SR, Reddy VY. Catheter Ablation of Atrial Fibrillation in Patients With Heart Failure: A Meta-analysis of Randomized Controlled Trials. Ann Intern Med 2018.

5. Anselmino M, Matta M, D’Ascenzo F, Bunch TJ, Schilling RJ, Hunter RJ, Pappone C, Neumann T, Noelker G, Fiala M, Bertaglia E, Frontera A, Duncan E, Nalliah C, Jais P, Weerasooriya R, Kalman JM, Gaita F. Catheter ablation of atrial fibrillation in patients with left ventricular systolic dysfunction: a systematic review and meta-analysis. Circ Arrhythm Electrophysiol 2014;7 (1941-3084 (Electronic)):1011-1018.

6. Prabhu S, Taylor AJ, Costello BT, Kaye DM, McLellan AJA, Voskoboinik A, Sugumar H, Lockwood SM, Stokes MB, Pathik B, Nalliah CJ, Wong GR, Azzopardi SM, Gutman SJ, Lee G, Layland J, Mariani JA, Ling LH, Kalman JM, Kistler PM. Catheter Ablation Versus Medical Rate Control in Atrial Fibrillation and Systolic Dysfunction: The CAMERA-MRI Study. J Am Coll Cardiol 2017;70 (16):1949-1961.

7. Prabhu S, Ling LH, Ullah W, Hunter RJ, Schilling RJ, McLellan AJ, Earley MJ, Sporton SC, Voskoboinik A, Blusztein D, Mariani JA, Lee G, Taylor AJ, Kalman JM, Kistler PM. The Impact of Known Heart Disease on Long-Term Outcomes of Catheter Ablation in Patients with Atrial Fibrillation and Left Ventricular Systolic Dysfunction: A Multicenter International Study. J Cardiovasc Electrophysiol 2016;27 (3):281-9.

8. Marrouche NF, Brachmann J, Andresen D, Siebels J, Boersma L, Jordaens L, Merkely B, Pokushalov E, Sanders P, Proff J, Schunkert H, Christ H, Vogt J, Bansch D, Investigators C-A. Catheter Ablation for Atrial Fibrillation with Heart Failure. N Engl J Med 2018;378 (5):417-427. 
9. Hunter RJ, Berriman TJ, Diab I, Kamdar R, Richmond L, Baker V, Goromonzi F, Sawhney V, Duncan E, Page SP, Ullah W, Unsworth B, Mayet J, Dhinoja M, Earley MJ, Sporton S, Schilling RJ. A randomized controlled trial of catheter ablation versus medical treatment of atrial fibrillation in heart failure (the CAMTAF trial). Circ Arrhythm Electrophysiol 2014;7 (1):31-8.

10. Jones DG, Haldar SK, Hussain W, Sharma R, Francis DP, Rahman-Haley SL, McDonagh TA, Underwood SR, Markides V, Wong T. A randomized trial to assess catheter ablation versus rate control in the management of persistent atrial fibrillation in heart failure. J Am Coll Cardiol 2013;61 (18):1894-903.

11. Khan MN, Jais P, Cummings J, Di Biase L, Sanders P, Martin DO, Kautzner J, Hao S, Themistoclakis S, Fanelli R, Potenza D, Massaro R, Wazni O, Schweikert R, Saliba W, Wang P, Al-Ahmad A, Beheiry S, Santarelli P, Starling RC, Dello Russo A, Pelargonio G, Brachmann J, Schibgilla V, Bonso A, Casella M, Raviele A, Haissaguerre M, Natale A. Pulmonary-vein isolation for atrial fibrillation in patients with heart failure. N Engl J Med 2008;359 (17):1778-85.

12. Di Biase L, Mohanty P, Mohanty S, Santangeli P, Trivedi C, Lakkireddy D, Reddy M, Jais P, Themistoclakis S, Dello Russo A, Casella M, Pelargonio G, Narducci ML, Schweikert R, Neuzil P, Sanchez J, Horton R, Beheiry S, Hongo R, Hao S, Rossillo A, Forleo G, Tondo C, Burkhardt JD, Haissaguerre M, Natale A. Ablation Versus Amiodarone for Treatment of Persistent Atrial Fibrillation in Patients With Congestive Heart Failure and an Implanted Device: Results From the AATAC Multicenter Randomized Trial. Circulation 2016;133 (17):1637-44.

13. Sugumar H, Prabhu S, Voskoboinik A, Young S, Gutman SJ, Wong GR, Parameswaran R, Nalliah CJ, Lee G, McLellan AJ, Taylor AJ, Ling LH, Kalman JM, Kistler PM. Atrial Remodeling Following Catheter Ablation for Atrial Fibrillation-Mediated Cardiomyopathy: Long-Term Follow-Up of CAMERA-MRI Study. JACC Clin Electrophysiol 2019;5 (6):681-688.

14. Prabhu S, Costello BT, Taylor AJ, Gutman SJ, Voskoboinik A, McLellan AJA, Peck KY, Sugamar H, Iles L, Pathik B, Nalliah CJ, Wong GR, Azzopardi SM, Lee G, Mariani J, Kaye DM, Ling L-H, Kalman JM, Kistler PM. Regression of Diffuse Ventricular Fibrosis Following Restoration of Sinus Rhythm With Catheter Ablation in Patients With Atrial Fibrillation and Systolic Dysfunction. JACC: Clinical Electrophysiology 2018:682.

15. Kuck K-H, Brugada J, Furnkranz A, Metzner A, Ouyang F, Chun KRJ, Elvan A, Arentz T, Bestehorn K, Pocock SJ, Albenque J-P, Tondo C. Cryoballoon or Radiofrequency Ablation for Paroxysmal Atrial Fibrillation. New England Journal of Medicine 2016;374 (23):2235-2245.

16. Pruszkowska P, Lenarczyk R, Gumprecht J, Jedrzejczyk-Patej E, Mazurek M, Kowalski O, Sokal A, Podolecki T, Morawski S, Streb W, Mitrega K, Kalarus Z. Cryoballoon ablation of atrial fibrillation in patients with advanced systolic heart failure and cardiac implantable electronic devices. Kardiol Pol 2018;76 (7):1081-1088.

17. Providencia R, de Asmundis C, Chun J, Chierchia G, Defaye P, Anselme F, Creta A, Lambiase PD, Schmidt B, Chen S, Hunter RJ, Combes S, Honarbakhsh S, Combes N, Sousa MJ, Jebberi Z, Albenque J-P, Boveda S. Catheter ablation of atrial fibrillation in patients with heart failure with reduced ejection fraction: Real world experience from six European centers. Journal of Cardiovascular Electrophysiology 2019;30 (8):1270-1277.

18. Voskoboinik A, Moskovitch JT, Harel N, Sanders P, Kistler PM, Kalman JM. Revisiting pulmonary vein isolation alone for persistent atrial fibrillation: A systematic review and meta-analysis. Heart Rhythm 2017;14 (5):661-667.

19. Shao M, Shang L, Shi J, Zhao Y, Zhang W, Zhang L, Li Y, Tang B, Zhou X. The safety and efficacy of second-generation cryoballoon ablation plus catheter ablation for persistent atrial fibrillation: A systematic review and meta-analysis. PLoS One 2018;13 (10):e0206362. 
20. Straube F, Hartl S, Dorwarth U, Wankerl M, Bunz B, Ebersberger U, Hoffmann E. Cryoballoon ablation for persistent atrial fibrillation - Large single-center experience. J Cardiol 2016;68 (6):492-497.

21. Steinberg JS, O'Connell H, Li S, Ziegler PD. Thirty-Second Gold Standard Definition of Atrial Fibrillation and Its Relationship With Subsequent Arrhythmia Patterns: Analysis of a Large Prospective Device Database. Circ Arrhythm Electrophysiol 2018;11 (7):e006274.

22. Verma A, Jiang CY, Betts TR, Chen J, Deisenhofer I, Mantovan R, Macle L, Morillo CA, Haverkamp W, Weerasooriya R, Albenque JP, Nardi S, Menardi E, Novak P, Sanders P, Investigators SAI. Approaches to catheter ablation for persistent atrial fibrillation. N Engl J Med 2015;372 (19):1812-22.

23. Mann I, Sasikaran T, Sandler B, Babalis D, Johnson N, Falaschetti E, Copley A, Tayebjee M, Todd D, Shepherd E, McCready J, Poulter NF, Kanagaratnam P. Ablation versus Anti-Arrhythmic Therapy for Reducing All Hospital Episodes from Recurrent Atrial Fibrillation (AVATAR-AF): Design and rationale. Am Heart J 2019;214 :36-45.

Tables and Figures

Table 1: Baseline Characteristics

\begin{tabular}{|c|c|}
\hline $\mathrm{N}=206$ & CRYO $(n=76)$ \\
\hline Age (years) & $63 \pm 11$ \\
\hline Gender (\% female) & $20 \%$ \\
\hline Hypertension (\%) & $21 \%$ \\
\hline Diabetes $(\%)$ & $10.5 \%$ \\
\hline Ischaemic HD (\%) & $37 \%$ \\
\hline Average $\mathrm{CHADS}_{2}$ VASC score & $2.66 \pm 1.40$ \\
\hline Average follow-up (months) & $28.1 \pm 12.9$ \\
\hline Continuous monitoring (\%) & $34 \%$ \\
\hline PPM (\%) & $1.3 \%$ \\
\hline $\operatorname{ICD}(\%)$ & $14 \%$ \\
\hline $\mathrm{BiV}(\%)$ & $17 \%$ \\
\hline Implanted loop recorder & $1.3 \%$ \\
\hline Pre ablation LVEF & $34 \pm 8.7 \%$ \\
\hline $\operatorname{DCM}(\%)$ & $47 \%$ \\
\hline Ischaemic CM (\%) & $37 \%$ \\
\hline Valvular (\%) & $5.2 \%$ \\
\hline Hypertrophic (\%) & $0 \%$ \\
\hline Other CM (\%) & $17 \%$ \\
\hline LA diameter & $46 \pm 6.5$ \\
\hline Paroxysmal AF (\%) & $29 \%$ \\
\hline $\begin{array}{l}\text { Persistent AF (\%) (Including long standing } \\
\text { persistent) }\end{array}$ & $71 \%$ \\
\hline Long-standing persistent AF (\%) & $26 \%$ \\
\hline Average NYHA Class & $2.37 \pm 0.72$ \\
\hline Medications & Medications \\
\hline Beta-blocker (\%) & $78 \%$ \\
\hline ACE Inhibitor or ARB (\%) & $91 \%$ \\
\hline Spirinolactone $(\%)$ & $10 \%$ \\
\hline AAD therapy (\%) & $54 \%$ \\
\hline AAD therapy + Beta blocker (\%) & $89 \%$ \\
\hline Amiodarone (\%) & $41 \%$ \\
\hline Sotalol (\%) & $7.9 \%$ \\
\hline Flecanide (\%) & $3.9 \%$ \\
\hline
\end{tabular}




\begin{tabular}{ll}
\hline $\mathbf{N}=\mathbf{2 0 6}$ & CRYO $(\mathbf{n}=\mathbf{7 6})$ \\
\hline Vitamin K antagonist (\%) & $32 \%$ \\
NOAC (\%) & $68 \%$ \\
\hline
\end{tabular}

\begin{tabular}{ll}
\hline $\mathbf{N}=\mathbf{2 0 6}$ & $\mathbf{R F}(\mathbf{n}=\mathbf{1 3 0})$ \\
\hline PVI isolation achieved & $99 \%$ \\
Cavo-tricuspid Isthmus ablation & $18 \%$ \\
Freeze time (mins) & $19.6 \pm 8.0$ \\
Procedure time & $90 \pm 42$ \\
DAP (mGy/cm ${ }^{\mathbf{3}}$ ) & $470 \pm 715$ \\
Repeat ablation & $13 \%$ \\
Average number of procedures & $1.17 \pm 0.37$ \\
\hline
\end{tabular}

Table 2: Procedural Characteristics

Table 3: Complications

\begin{tabular}{ll}
\hline $\mathbf{N}=\mathbf{2 0 6}$ & $\mathbf{C R Y O}(\mathbf{n}=\mathbf{7 6})$ \\
\hline Cardiac tamponade & $0 \%$ \\
Stroke / TIA* & $0 \%$ \\
Vascular complication* & $2.2 \%(2)$ \\
Phrenic nerve injury & $0 \%$ \\
Oesophageal injury*** & $1.3 \%(1)$ \\
\hline
\end{tabular}

*Intra-procedural or within 30 days post procedure

**Requiring intervention or blood transfusion.

*** Occurred following repeat procedure with $\mathrm{RF}$ ablation.

Table 4 Long term clinical success

\begin{tabular}{llll}
\hline $\mathbf{N}=\mathbf{7 6}$ & 1 year single procedure success & Long term single procedure success & Long \\
\hline Overall & $70 \%$ & $43 \%$ & $59 \%$ \\
Off AAD (\%) & $41 \%$ & $30 \%$ & $40 \%$ \\
Paroxysmal AF & $81 \%$ & $50 \%$ & $64 \%$ \\
Persistent AF & $65 \%$ & $41 \%$ & $57 \%$ \\
Long-standing persistent AF & $29 \%$ & $21 \%$ & $50 \%$ \\
\hline
\end{tabular}

Supplementary materials

Table 1 - Cox regression analysis of predictors of single procedural success

\begin{tabular}{|c|c|c|c|}
\hline Variable & $P$ value & 95\% CI lower value & 95\% CI upper value \\
\hline $\begin{array}{l}\text { CHADS }_{2} \text { VASc } \\
\text { Score }\end{array}$ & 0.506 & 0.554 & 3.313 \\
\hline NYHA Class & 0.131 & 0.774 & 7.152 \\
\hline
\end{tabular}




\begin{tabular}{llll} 
Baseline LVEF & 0.188 & 0.831 & 1.037 \\
Age & 0.247 & 0.961 & 1.168 \\
Ischaemic & 0.760 & 0.136 & 4.289 \\
cardiomyopathy & & & \\
$\begin{array}{l}\text { Paroxysmal AF } \\
\text { Long standing }\end{array}$ & 0.763 & 0.246 & 6.789 \\
persistent AF & 0.692 & 0.275 & 7.004 \\
Hypertension & 0.247 & 0.439 & 24.754 \\
Diabetes & 0.522 & 0.247 & 15.749 \\
Baseline LA area & 0.824 & 0.911 & 1.124 \\
\hline
\end{tabular}

\section{Legends for Figures}

Figure 1

A Kaplan Meier curve illustrating the single procedure freedom from AF recurrence for patients undergoing index cryoablation over they study period, stratified via AF phenotype.

\section{Figure 2}

Improvement in absolute left ventricular ejection fraction (panel A) and NYHA functional class (panel B) from baseline and follow up. NYHA = New York Heart Association.

\section{Hosted file}

Cryo paper Figures Final.pptx available at https://authorea.com/users/365062/articles/485319long-term-outcomes-of-index-cryo-balloon-ablation-in-patients-with-atrial-fibrillationand-systolic-heart-failure 